Apidologie, 1972, 3 (2), 149-165.

\title{
OBSERVATIONS PRÉLIMINAIRES SUR LA BIOLOgie D'OSMIA (CHALCOSMIA) CERULESCENS L., (HYMENOPTERA MEGACHILIDAE), POLLINISATRICE DE LA LUZERNE (MEDICAGO SATIVA L.)
}

Vorläufige Beobachtungen über die Biologie der die Luzerne bestäubenden Osmia (Chalcosmia) coerulescens $L$. (Hymenoptera Megachilidae)

\author{
Jean-Noël TASEI \\ avec la collaboration technique de Murielle Picant \\ Laboratoire d'Ethologie et d'Écologie des Insectes, I.N.R.A., \\ 86-Lusignan
}

\section{SUMMARY}

\author{
PReliminary observations on the blology of Osmia (Chalcosmia) \\ corulescens L. (hymenoptera, megachilidae) \\ POLLINATING LUCERNE (Medicago sativa L.)
}

During three years the author investigated the biology of an apoid Megachilidae, common in the district of Lusignan : Osmia coerulescens L. This Osmia can sometimes be seen on lucerne flowers.

The pollen analysis of nests provisions shows that this bee collects pollen on Labiatae and chiefly on Papilionaceae : Trifolium, Medicago sativa, Lotus etc... O. coerulescens has one or two generations per year, extending from May to September. In greenhouse a female can lay about fourty eggs. At constant $21^{\circ} \mathrm{C}$, the adult appears 6 or 7 weeks after the egg laying.

The bee nests in holes of timber and old buildings facing east or south. Nest's partitions are built with a paste of chewed plants (Malva moschata).

It could be possible to increase Osmia populations by settling artificial nesting sites and move them in fields of lucerne sown for seed. 


\section{RÉSUMÉ}

L'auteur fait part de résultats concernant trois années d'observation d'un apoïde $\mathbf{M e g a -}$ chilidae : Osmia coerulescens $L$., fréquent dans la région de Lusignan. Cette osmie se rencontre parfois sur les fleurs de luzerne cultivée pour la graine. L'analyse des provisions de nids révèle que l'abeille récolte du pollen de labiées, mais surtout de papilionacées : Trifolium, Medicago sativa, Lotus etc... O. coerulescens a une ou deux générations par an, de Mai à Septembre. En serre une femelle pond une quarantaine d'œufs. Le développement s'effectue en 6 ou 7 semaines à la température constante de $21^{\circ} \mathrm{C}$.

L'abeille nidifie dans des trous de charpentes et de vieux murs exposés à l'Est et au Sud. Les cloisons sont édifiées avec une pâte d'origine végétale (Malva moschata). On pourrait envisager d'établir des sites artificiels de nidification afin d'accroître les populations de cette osmie et de les déplacer dans des champs de luzerne cultivée pour la graine.

\section{INTRODUCTION}

Divers inventaires de la faune pollinisatrice de la luzerne ont été établis dans plusieurs pays. Les insectes les plus efficaces sont des Apoidea solitaires, fouisseurs et terricoles, de loin les plus abondants, et des Apoidea occupant des cavités du sol, des vieux murs et vieilles charpentes (LinsLey - 1946; Lesins - 1950; Bohart - 1957; Moczar - 1961; Lecomte et Tirgari - 1962; Obrtel et Sedivy - 1965; Tasei - 1970; Dylewska - 1970).

Trois espèces ont attiré l'attention des chercheurs :

Nomia melanderi Cock. (Halictidae) (Hackwell - 1968).

Melitta leporina Panz. (Melittidae) (TIrgari - 1968).

Megachile rotundata Fabr. (Megachilidae) (TIRGARI - 1968).

Ces études biologiques, entre autres, ont permis d'accroître artificiellement les populations de $N$. melanderi et $M$. rotundata, grâce à l'établissement de sites favorables à leur nidification (STEPHen - 1965, WALstrom et Jones 1968).

Comme un grand nombre de Megachilidae, $M$. rotundata peut loger dans des tubes de carton ou de bois. L'aménagement des sites est moins complexe que dans le cas de l'espèce terricole $N$. melanderi. Il nous a paru avantageux de rechercher parmi les Megachilidae pollinisateurs de la luzerne en France, une espèce nidifiant dans des nichoirs aériens comme $M$. rotundata .

En France on a rencontré 6 espèces de Megachilidae : Megachile (Eutricharea) leachella, M. centuncularis, Osmia aurulenta, O. rufohirta, O. corulescens (Taser non publié) et Anthidium punctatum (Lecomte et Tirgari 1962).

La seule espèce qui occupe en grand nombre les pièges nichoirs que nous proposons aux Megachilidae, est $O$. cœrulescens. 
Il est bon de noter que le genre Osmia figure très rarement dans les inventaires de pollinisateurs de la luzerne : BoHART (1957) signale Osmia seclusa Sandhouse et Diceratosmia subfasciata punctata Mich. OBRTEL et Sedvy (1965) une espèce non déterminée et Iuga et Scobiola (1960) O. cephalotes Mor ${ }^{1}$ et Solinas et Bin (1965) O. aenea $\mathrm{F}^{2}$.

A notre connaissance $O$. corulescens n'a jamais été l'objet d'études détaillées. Les auteurs cités dans la bibliographie donnent des indications brèves sur la structure du nid, ou les plantes butinées. Nous avons eu l'occasion d'observer cette espèce pendant 3 années consécutives. En 1969, O. cœrulescens a nidifié dans un nichoir de bois placé dans le Moyen Atlas (Maroc), et dans le courant du mois de juillet, nous avons remarqué plusieurs femelles en train de polliniser des fleurs de luzerne dans la région de Rabat. En 1970 et 1971, dans la région de Lusignan, où cette osmie est très commune, nous avons recueilli de nombreux renseignements sur le choix du site, la construction, l'approvisionnement du nid, le comportement de butinage et le développement d'O. corulescens.

\section{MATÉRIEL ET MÉTHODES}

\section{I. - Le dispositif d'observation par « piège-nichoirs 》}

En 1971 nous avons placé dans 7 localités ${ }^{3}$ proches de Lusignan, 55 nichoirs de bois $(9,5 \times 9,5 \times 15 \mathrm{~cm})$. Chaque nichoir (Fig. 1) est pourvu de 10 tubes en carton de $4 \mathrm{~mm}$ de diamètre et $55 \mathrm{~mm}$ de longueur et de 10 Cannes de Provence (Arundo L.) de $75 \mathrm{~mm}$ de longueur. Cinq sont percées de trous de $5 \mathrm{~mm}$ de diamètre, cinq autres sont percées de trous de $8 \mathrm{~mm}$. Toutes sont fendues en 2 parties longitudinales. Ce principe de nichoir est décrit par Leviv (1957).

Les localités sont dans des fonds de vallée ou bien sur le plateau de Lusignan (altitude : $150 \mathrm{~m}$ ). Dans chacune d'elles on répartit les nichoirs contre des arbres, des charpentes en bois, des bâtiments modernes crépis, des bâtiments en pierres non crépis. A chacune de ces places nous orientons 4 nichoirs dans 4 directions : un vers l'Est, un vers le Sud, un vers l'Ouest, un vers le Nord. Les nichoirs sont accrochés aux parois de leur support à une hauteur d'l à $2 \mathrm{~m}$. Ce dispositif est mis en place au début du mois d'avril et il est visité par la suite une fois tous les 7 jours. A chaque visite on note la présence des adultes mâles ou femelles à l'intérieur des tubes, le début de constructions, le nombre de cellules dans chaque nid. Tous les nids entièrement achevés par les femelles sont retirés, et leur place est occupée à nouveau par un tube vide. Chaque nid porte un numéro de référence qui indique la localité où il a été construit, l'emplacement, l'orientation, le diamètre et la place dans le nichoir.

\section{II. - L'élevage en enceinte fermée}

Afin de compléter les observations par “ piège-nichoirs » dans la nature il est bon de contraindre des femelles à nidifier dans des conditions contrôlées. Nous avons ainsi élevé en 1970 et 1971, 14 femelles dans des compartiments de serre vitrée $(3 \times 2,5 \mathrm{~m})$ possédant un toit en pente, des volets et une porte grillagée. Aucune température excessive n'a été enregistrée dans la serre (Maximum absolu : $31^{\circ} \mathrm{C}$, minimum absolu : $9^{\circ} \mathrm{C}$ ). Un nichoir portant des

1. L'auteur roumain ne précise pas le genre d'activité de cette espèce : récolte de nectar ou récolte de pollen.

2. Synonyme d'O. coerulescens $L$.

3. Par localité on entend : une ferme ou un hameau. 

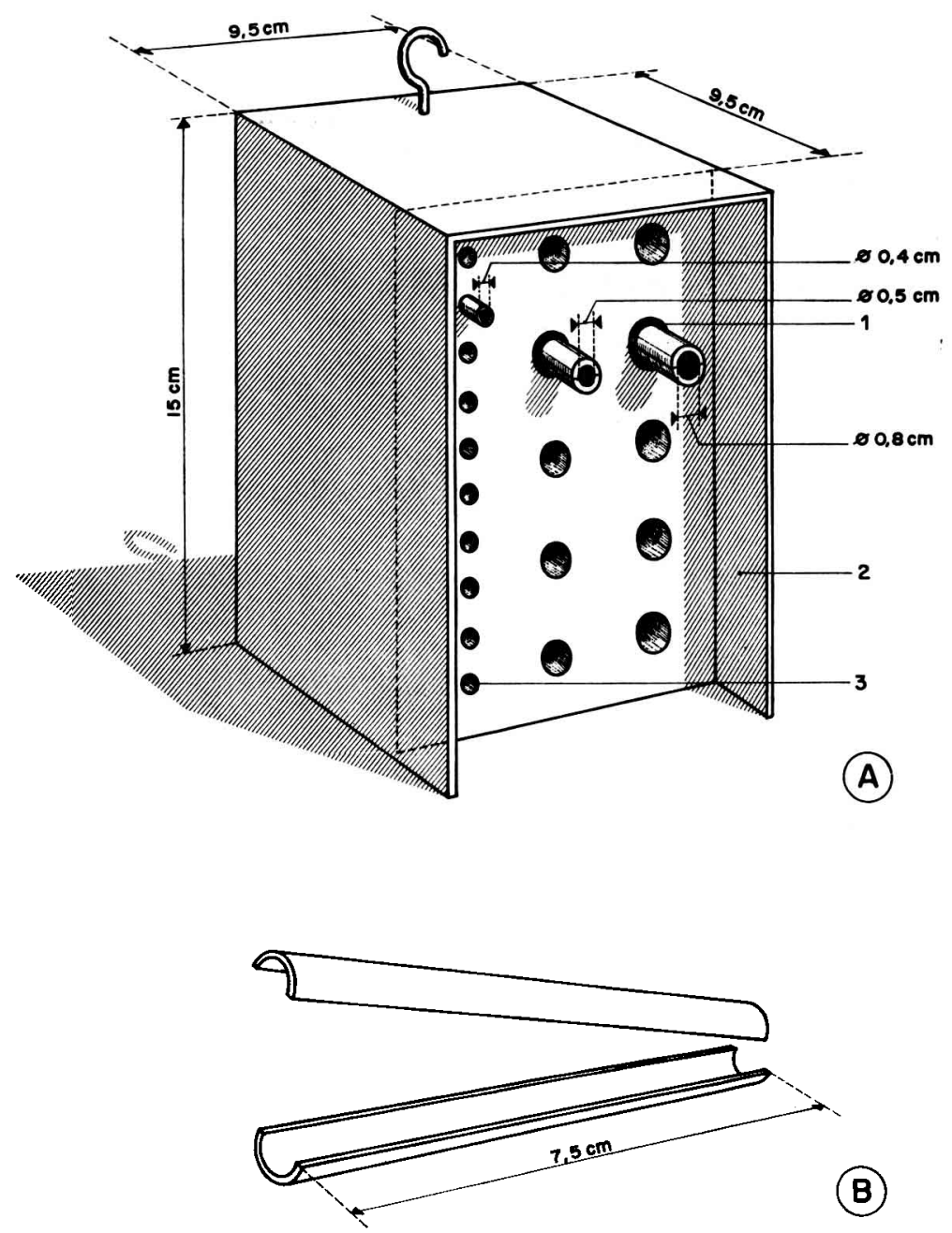

FIg. 1. - Piège-nichoir.

A. Vue d'ensemble. - 1. Bracelet de caoutchouc maintenant les deux moitiés d'un tube en canne de Provence. - 2. Auvent. - 3. Rangée de tubes de carton.

B. Tube de nidification (canne de Provence) extrait du nichoir.

\section{Aвв. 1. - Fallennest.}

A. Gesamtansicht. - 1. Gummiring, der die beiden Abschnitte des Rohrstengels stützt. - 2. Schutzdach. - 3. Papphülsen-Reihe.

B. Niststengel (Arundo L.) aus der Nistanlage herausgezogen.

tubes de carton de $4 \mathrm{~mm}$ de diamètre est disposé à $50 \mathrm{~cm}$ du sol, et des plantes fleuries en pot servent aux femelles pour approvisionner leurs cellules. Les abeilles sont introduites lorsqu'elles ont commencé à construire un nid à l'extérieur. Cette méthode permet à tous les individus de nidifier très rapidement après leur introduction en serre. Chaque jour tous les tubes sont visités. Dès qu'une femelle commence la construction d'un nid, elle est marquée d'une tache de peinture sur le thorax. Les tubes operculés sont retirés et portés au laboratoire. Les activités de construction et de butinage font l'objet d'observations détaillées tout au long de la nidification. 


\section{II. - La détermination des pollens récoltés dans la nature}

A chaque visite hebdomadaire du dispositif de nichoirs, on prélève un échantillon de " pain d'abeille " dans la première et la dernière cellule construites dans les nids operculés. On a ainsi effectué 130 prélèvements. On trouvera dans le travail de Maurizio et LouveauX (1965) la méthode de montage des préparations de pollens.

L'analyse microscopique des provisions des cellules d'osmie nous renseigne sur les plantes butinées pour le pollen, les dates de récolte des divers pollens, l'influence de la localité sur le spectre pollinique de la récolte.

\section{IV. - Les conditions d'élevage des aufs et des larves}

Les nids sont placés individuellement dans des tubes de plastique dont les deux extrémités sont percées et munies d'un grillage de laiton (4 mailles au mm). L'aération est ainsi assurée, et l'on empêche la prolifération d'un ectoparasite redoutable à cause de son cycle de développement très court (BALFour-Browne F. 1922) : Melittobia acasta Walk. (Hymenoptera, Eulophidae). Une partie de l'élevage est conservée dans un abri à l'extérieur du laboratoire, l'autre partie poursuit son développement à une température constante $\left(21^{\circ} \mathrm{C}, 28^{\circ} \mathrm{C}, 32^{\circ} \mathrm{C}\right)$ et dans une atmosphère dont l'humidité relative est maintenue entre 60 et $80 \%$. Le développement des insectes en étuve est contrôlé tous les jours à la même heure. Le passage d'un stade larvaire à un autre est difficile à saisir; aussi on saupoudre très légèrement les larves avec de la terre finement pulvérisée. Ce saupoudrage permet de connaître de façon très sûre le moment de la mue car il est facile de repérer l'élimination de la terre lorsque l'exuvie se détache du corps de la larve. Après chaque mue on effectue un nouveau saupoudrage etc... Lorsque les larves ont filé leur cocon, l'observateur doit pratiquer une ouverture dans l'enveloppe de soie, afin de suivre l'évolution de l'individu (Transformation de la larve en nymphe et de la nymphe en adulte).

\section{RESULTATS}

\section{I. - Comportement de nidification}

1. Les périodes de vol.

$O$. cœrulescens peut nidifier de fin avril à fin septembre. D'après Friese (1911) et Benorst (1931) cette espèce vole en mai et juin. En réalité il y a 4 mois de grande activité : mai, juin, juillet, août, et c'est en juin que le plus grand nombre de femelles nidifie (fig. 2). Il semble que seules les températures supérieures à $19-20^{\circ} \mathrm{C}$ permettent aux osmies de voler et d'approvisionner les nids. En serre, si les conditions atmosphériques sont favorables, les femelles accomplissent 10 à 12 heures de travail par jour.

\section{L'architecture du nid et le comportement de construction.}

Les nids observés sont formés par des cellules disposées linéairement. Les cloisons qui séparent les cellules sont élevées par la femelle (fig. 3). Celleci mâche des morceaux de végétaux et les imbibe de salive, puis édifie les cloisons qui prennent en séchant une couleur vert noirâtre. En serre ce ciment végétal est fabriqué à partir de boutons de fleurs d'Onobrychis et de Medicago sativa, de calice et de feuilles de Papaver et des folioles de Lotus. Cependant, 


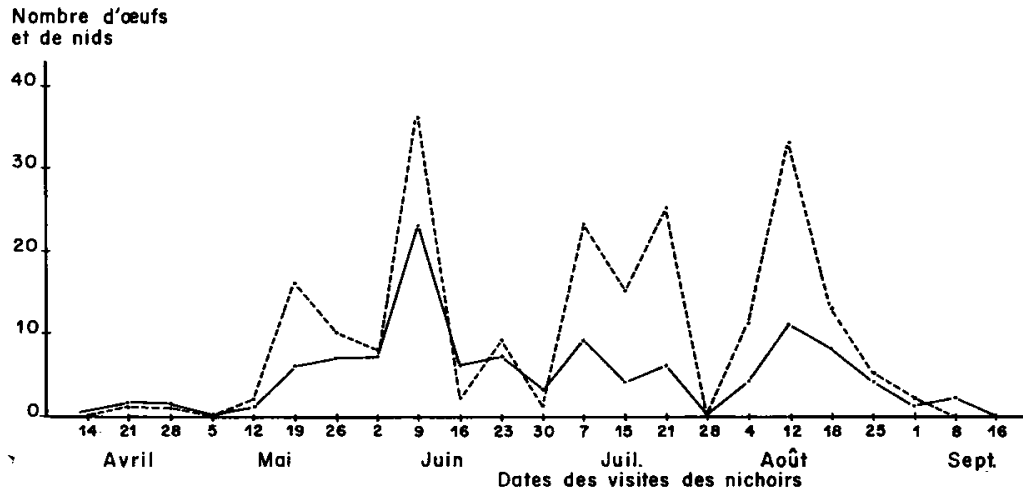

Frc. 2:-Activitê de nidification d'O. coerulescens en 1971 à Lusignan. Axe des $\mathrm{x}$ : dates des visites des nichoirs. Axe des y : nombre d'cufs pondus entre 2 visites consécutives (trait pointillé). Nombre de Lnids en cours de construction (trait plein).

Aв8. 2. - Nestbau von Osmia coerulescens L. 1971 in Lusignan.

x-Achse : Nachschaudaten.

y-Achse : Zahl der zwischen zwei aufeinanderfolgenden Nachschauen gelegten Eier. Zahl der im Bau befindlichen Nester.

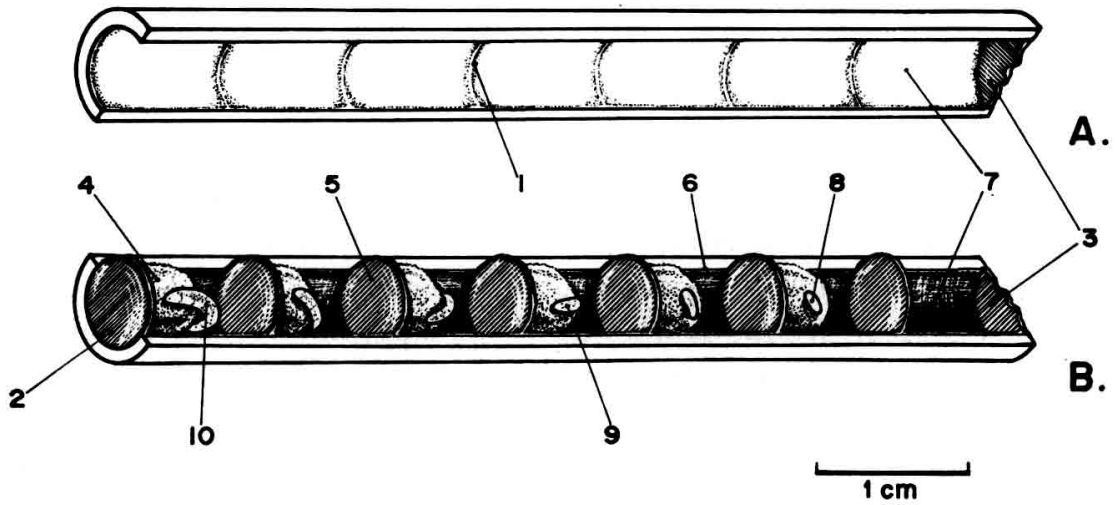

Fic. 3. - Nid d'O. coerulescens dans une canne de Provence.

1. Trace des cloisons. - 2. Bouchon initial. - 3. Bouchon terminal. -4 . Provision de pollen. -5 . Cloison en ciment d'origine végétale. - 6. Dépôt de ciment végétal fait par la femelle à la jointure des deux parties du tube. -7 . Vestibule. -8 , ouf. -9 . Jeune larve. -10 . Larve de $4^{e}$ stade.

Aвв. 3. - Nest von Osmia coerulescens in einem Rohrstengel.

1. Umriss der Scheidewände. - 2. Anfangsverschluss. - 3. Endverschluss. - 4. Pollenvorrat. - 5. Verschlusspropfen aus Mörtel pflanzlichen Ursprungs. - 6. Vom Weibchen am Gelenk der Stengelabschnitte abgelegter pflanzlicher Mörtel. - 7. Vorraum. - 8. Ei. - 9. junge Larve. - 10. Larve des 4. Stadiums. 
le végétal de loin le plus attractif est Malva moschata $L$. (calice et feuilles). Il arrive qu'en l'absence de cette espèce, l'osmie éprouve de la difficulté à construire. Une telle spécificité dans le choix du matériau de construction se rencontre chez Osmia lanosa (Ferton - 1894). Cependant Ghandeer (1963) cite les genres Leonurus et Nepeta et Friese (1911) Alnus glutinosa, comme matériaux de construction pour $O$. cærulescens. Nous avons observé la construction d'une cloison en $20 \mathrm{~mm}$ et celle d'un bouchon terminal en $48 \mathrm{~mm}$. La cloison a demandé 8 allers et retours de la mauve au nid, le bouchon terminal : 16. La femelle passe en moyenne deux fois plus de temps à déposer la bouillie végétale dans le nid, qu'à prélever les tissus de la plante. Pendant la construction de la cloison la femelle n'a pas butiné une seule fleur, alors qu'au cours de la construction du bouchon terminal elle a passé plus de $5 \mathbf{~ m n}$ sur des fleurs de Melilotus et Lotus. Comme la plupart des osmies, O. corrulescens édifie un " pas de porte » avant d'approvisionner une cellule. Ce " pas de porte $\gg$ est l'amorce de la future cloison, qui fermera la cellule après la ponte; il peut être réduit à une trace de bouillie végétale, formant un anneau vert sur la paroi du tube.

Toutes les cellules sont approvisionnées, mais dans $80 \%$ des cas la dernière est vide (vestibule). Les cloisons sont très fines (moins de $0,5 \mathrm{~mm}$ ) mais leur épaisseur augmente à l'endroit où elles se rattachent au tube. Leur concavité est tournée vers la sortie. Le bouchon terminal est plus épais que les cloisons (1 à $5 \mathrm{~mm}$ ) et il est fait de la même matière que celles-ci. La longueur des cellules varie assez peu pour un diamètre donné. En moyenne pour un diamètre de $4 \mathrm{~mm}$ la longueur d'une cellule est $8,5 \mathrm{~mm}$; pour un diamètre de $7 \mathrm{~mm}$ : $6,5 \mathrm{~mm}$. Les vestibules ont des longueurs très variables de 6 à $70 \mathrm{~mm}$. $O$. corulescens occupe généralement toute la place qui lui est offerte et les nids contiennent souvent 6 à 8 cellules (tubes de $84 \mathrm{~mm}$ de longueur). On a même obtenu un nid de 15 cellules dans un tube de $104 \mathrm{~mm}$ de longueur. Une femelle peut fonder 5 à 10 nids au cours de sa vie. Un seul auteur : Malyshev (1935) relate l'existence de nids formant des “ pseudorayons 》, composés de cellules disposées côte à côte sur un même plan.

\section{La ponte.}

Une femelle captive pond en moyenne 42 aufs au cours de sa vie en serre. Ce résultat concerne les années 1970 et 1971 (591 œufs). Suivant les lots de femelles, un individu pond de 31 à 50 aufs. Dans les conditions naturelles une femelle pond 1 à 2 aufs par jour; en serre elle pond 1 à 6 oufs. La ponte moyenne journalière en serre par femelle est 3,4 ceufs. (Résultat concernant 119 aufs). Les nids peuvent contenir seulement des cellules de mâles ou bien 
seulement des cellules de femelles, ou encore les deux sortes de cellules, celles de mâles étant les plus proches de la sortie. Le sex-ratio est très variable : dans les conditions de la serre en 1970 nous avons obtenu en première génération 1 femelle pour 1,1 mâle; en 1971 : 1 femelle pour 5 mâles, et dans les conditions naturelles en 1971 : 1 femelle pour 0,6 mâle.

\section{La longévité.}

En serre les femelles vivent de 30 à 60 jours. Lorsqu'elles vieillissent, la confection des charges de pollen demande beaucoup plus de temps et les derniers jours de leur vie ne sont plus employés à approvisionner des cellules, mais à construire des cloisons ou des bouchons de manière anarchique. Les femelles meurent généralement hors du nid.

\section{Le choix du site.}

Les nichoirs sont très inégalement occupés : $67 \%$ d'entre eux n'ont pas abrité de nidification, $22 \%$ renfermaient 2 à 8 nids, $11 \% 1$ seul nid. $O$. cœrulescens préfère fonder ses nids dans des tubes orientés vers l'Est ou le Sud. Les nichoirs occupés par les osmies et exposés à l'Est et au Sud contiennent respectivement 3,1 et 2,1 nids par nichoir, tandis que ceux qui sont exposés au Nord et à l'Ouest contiennent seulement 0,6 et 0,5 nid par nichoir. Les expositions au Nord et à l'Ouest favorisent les abandons de nid, avant même la ponte du premier œuf (cf. tableau 1).

Les tubes de $4 \mathrm{~mm}$ de diamètre abritent $64 \%$ des nids, les tubes de $5 \mathrm{~mm}$ : $33 \%$, les tubes de $8 \mathrm{~mm}: 3 \%$. Si l'on admet que la population d'un nichoir est représentative de la population locale, il apparaît que cette osmie nidifie de préférence dans des bâtiments en bois. A ces emplacements les nichoirs portent en moyenne 4,3 nids par nichoir. Les constructions en pierres non crépies sont moins favorables (1,5 nid par nichoir). Les constructions modernes crépies et les haies vives sont très peu favorables aux nidifications $(0,4$ et 0,3 nid par nichoir).

Friese (1923) relate la découverte de nids d'O. corulescens dans de vieux poteaux. Il affirme que les cloisons sont en argile. BENorst (1938) décrit une nidification $d^{\prime} O$. coerulescens dans une branche de sapin creusée par un coléoptère xylophage et MARIKovskayA (1968) signale des nidifications de cette espèce dans des terriers d'Osmia jagnobensis F. D'après Krombein (1967) et Medler (1967) les diamètres des nids s'échelonnent de 4,8 à 6,4 mm. Free et Williams (1970) notent qu'O. coerulescens a tendance à nidifier sur les lieux de sa naissance. 
6. Le butinage et l'approvisionnement du nid.

a) Le butinage en serre.

Onze familles de plantes, représentées par 25 espèces, sont proposées aux femelles :

- Boraginaceae : Myosotis sp.

- Umbelliferae : Heracleum sp.

- Papaveraceae : Papaver sp.

- Rubiaceae : Galium sp.

- Malvaceae : Malva moschata.

- Compositae : Sonchus asper, Lapsana communis, Pterotheca sp.

- Cruciferae : Sinapis sp.

- Ranunculaceae : Ranunculus acris.

- Hypericaceae : Hypericum perforatum.

- Labiatae: Salvia pratensis, Glechoma hederacea.

- Papilionaceae : Hedysarum sp., Onobrychis sativa, Melilotus alba, $M$. arvensis, Trifolium repens, T. pratense, T. hybridum, Medicago sativa, Vicia cracca, V. sepium, Lotus corniculatus, Coronilla varia.

Aucune des 9 premières familles n'est visitée par $O$. cœrulescens qui butine seulement sur Salvia, Glechoma et surtout sur les papilionacées (excepté Hedysarum). Melilotus alba est la plante la plus attractive. Les autres papilionacées sont en moyenne d'attractivité égale et très sensiblement inférieure à celle du mélilot. La rapidité de butinage est 10,3 à 11,3 fleurs par minute chez Lotus, Onobrychis, Coronilla; 13 à 14,2 fleurs par minute chez Trifolium et Melilotus; 9,7 à 10,2 fleurs par minute chez Medicago sativa. Pratiquement $100 \%$ des fleurs de luzerne visitées sont déclenchées. Les durées de voyages de butinage varient de 3 à 15 minutes (moyenne calculée sur 100 voyages : $9 \mathrm{~mm} \mathrm{24} \mathrm{s})$.

b) L'approvisionnement des cellules en serre.

Une cellule est approvisionnée en moyenne au bout de 3 heures de butinage. L'approvisionnement nécessite une vingtaine de voyages. Le poids moyen d'une provision de ( pain d'abeille ) dans une cellule est $75 \mathrm{mg}$ (93 cellules examinées). Les poids extrêmes sont 47 et $127 \mathrm{mg}$. La teneur en eau des provisions est $28 \%$ du poids frais. La femelle qui vient de garnir sa brosse entre dans son nid la tête la première et régurgite nectar et sécrétion salivaire pendant une vingtaine de secondes, puis elle sort du nid et y pénètre à nouveau à reculons pour décharger le pollen. Cette deuxième opération occupe la femelle 20 à 30 secondes. 
TABL. 1. - Choix du site de nidification en fonction de l'exposition.

TAB. 1. - Wahl des Nistplatzes in bezug auf die Himmelsrichtung

\begin{tabular}{|c|c|c|c|c|c|}
\hline $\begin{array}{c}\text { Exposition } \\
\text { Himmelsrichtung }\end{array}$ & $\begin{array}{c}\text { Est } \\
\text { Osten }\end{array}$ & $\begin{array}{l}\text { Sud } \\
\text { Suiden }\end{array}$ & $\begin{array}{l}\text { Ouest } \\
\text { Westen }\end{array}$ & $\begin{array}{l}\text { Nord } \\
\text { Norden }\end{array}$ & \\
\hline $\begin{array}{l}\text { Nombre de nichoirs proposés } \ldots \ldots \ldots \ldots \ldots \ldots \\
\text { Zahl d. angebotenen Nistplätze } \ldots \ldots \ldots \ldots \ldots \ldots\end{array}$ & 13 & 15 & 13 & 14 & 55 \\
\hline $\begin{array}{l}\text { Nombre de nichoirs occupés } \ldots \ldots \ldots \ldots \ldots \\
\text { Zahl d. angenommenen Nistplätze } \ldots \ldots \ldots \ldots \ldots \ldots\end{array}$ & 5 & 5 & 5 & $\mathbf{3}$ & 18 \\
\hline $\begin{array}{l}\text { Nombre de nids achevés } \ldots \ldots \ldots \ldots \ldots \ldots \ldots \\
\text { Zahl d. vollendeten Nester } \ldots \ldots \ldots \ldots \ldots \ldots \ldots \ldots\end{array}$ & 36 & 27 & 3 & 5 & 71 \\
\hline $\begin{array}{l}\text { Nombre de nids abandonnés } \ldots \ldots \ldots \ldots \ldots \\
\text { Zalh d. verlassenen Nester } \ldots \ldots \ldots \ldots \ldots \ldots \ldots\end{array}$ & 4 & 5 & 4 & 4 & 17 \\
\hline $\begin{array}{l}\text { Nombre total de nids par nichoir proposé..... } \\
\text { Gesamtzahl d. Nester je angebotenem Nistplatz..... }\end{array}$ & 3,1 & 2,1 & 0,5 & 0,6 & \\
\hline
\end{tabular}

c) Le butinage dans la nature.

L'analyse microscopique des provisions d'O. corulescens révèle que 14 espèces de plantes appartenant aux familles des labiées et des papilionacées, sont butinées pour le pollen. Les chiffres entre parenthèses accompagnant les noms de plantes ci-dessous, indiquent les fréquences de rencontre de chaque espèce de pollen, c'est-à-dire le nombre de préparations microscopiques (sur un total de 130), contenant un quelconque pourcentage de cette espèce :

- Papilionaceae : Trifolium repens (59), Medicago sativa (49), Trifolium pratense (49), Lotus corniculatus (46), Sarothamnus sp. (21), Onobrychis sativa (8), Vicia sp. (6).

- Labiatae : Calamintha clinopodium (26), Teucrium scorodonia (19), Stachys sp. (8), Brunella vulgaris (5), Ajuga reptans (5), Glechoma hederacea (2), Lamium purpureum (2).

Ces données confirment et précisent les résultats obtenus en serre : $O$. corulescens récolte le pollen de préférence sur les papilionacées. Les labiées doivent être considérées comme des plantes secondaires. Les quatre espèces les plus fréquemment visitées à Lusignan sont : Trifolium repens, Medicago sativa, Trifolium pratense, Lotus corniculatus. Si l'on s'en tient à la terminologie de Linsley (1958) et aux définitions de Robertson (1925). O. cœru- 
lescens est polylectique ${ }^{1}$, mais son comportement est très proche des espèces oligolectiques $^{2}$.

Les provisions sont constituées de une à cinq espèces de pollen. Le plus fréquemment on rencontre des mélanges de deux espèces. Les provisions uniflorales proviennent seulement de 6 espèces : Trifolium pratense, Sarothamnus, Trifolium repens, Medicago sativa, Lotus corniculatus, Onobrychis sativa. Les pollens de labiées sont mélangés à ceux des papilionacées surtout en début et fin de saison, lorsque les fleurs de trèfles, de luzerne et de lotier sont plus rares. Les spectres polliniques diffèrent d'une localité à une autre. Dans chacune apparaissent deux ou trois pollens dominants. Ces dominances sont probablement dues à l'abondance relative de deux ou trois plantes aux environs immédiats des nichoirs.

Peu de renseignements sur les plantes visitées par $O$. corulescens proviennent d'autres auteurs : Friese (1911) signale cette espèce sur Ajuga et Lotus; Cavro (1950) sur Philadelphus, Salvia, Trifolium et Vicia aux U.S.A., sur Malva, Lamium et Taraxacum en France; Iuga (1962) sur Prunus cesarus et Onobrychis sativa : Chandere (1963) sur Leonurus et Nepeta.

On peut penser que si $O$. corulescens nidifie à proximité d'une grande surface de luzerne, elle a tendance à visiter uniquement la luzerne. Dans cette hypothèse nous estimons le travail de pollinisation théorique d'une femelle au cours d'une journée. On suppose qu'un individu approvisionne une cellule par jour avec du pollen de luzerne, et déclenche 10 fleurs par minute. Cela représente 180 minutes de butinage, soit 1800 fleurs déclenchées par jour. En admettant que chaque gousse contienne 5,5 graines et que 1000 graines pèsent $2,4 \mathrm{~g}$, chaque femelle est responsable de la production de $23,7 \mathrm{~g}$ de graines de luzerne par jour. Tirgari (1968) estime par la même méthode qu'une femelle de Melitta leporina Panz. produit 5,7 g de graines par jour. En effet, d'après cet auteur, M. leporina n'approvisionne qu'une cellule par jour, et une grande habileté à récolter le pollen de luzerne lui permet de constituer la provision de pollen en 96 minutes de butinage. C'est donc une espèce moins efficace qu'O. coerulescens, bien que déclenchant la luzerne à la vitesse de 10 fleurs minute.

\section{II. - Développement}

\section{Les stades de développement. (Tableau 2).}

Nous donnons ici les résultats obtenus avec des individus mâles à diapause imaginale. A la température constante de $21^{\circ} \mathrm{C}$, le développement de l'œuf

1. polylectique : qui récolte le pollen sur des plantes appartenant à plusieurs familles botaniques.

2. oligolectique : qui récolte, de façon constante, le pollen sur une espèce de plante ou sur plusieurs espèces appartenant à la même famille botanique, même en présence d'autres sources de pollen. 


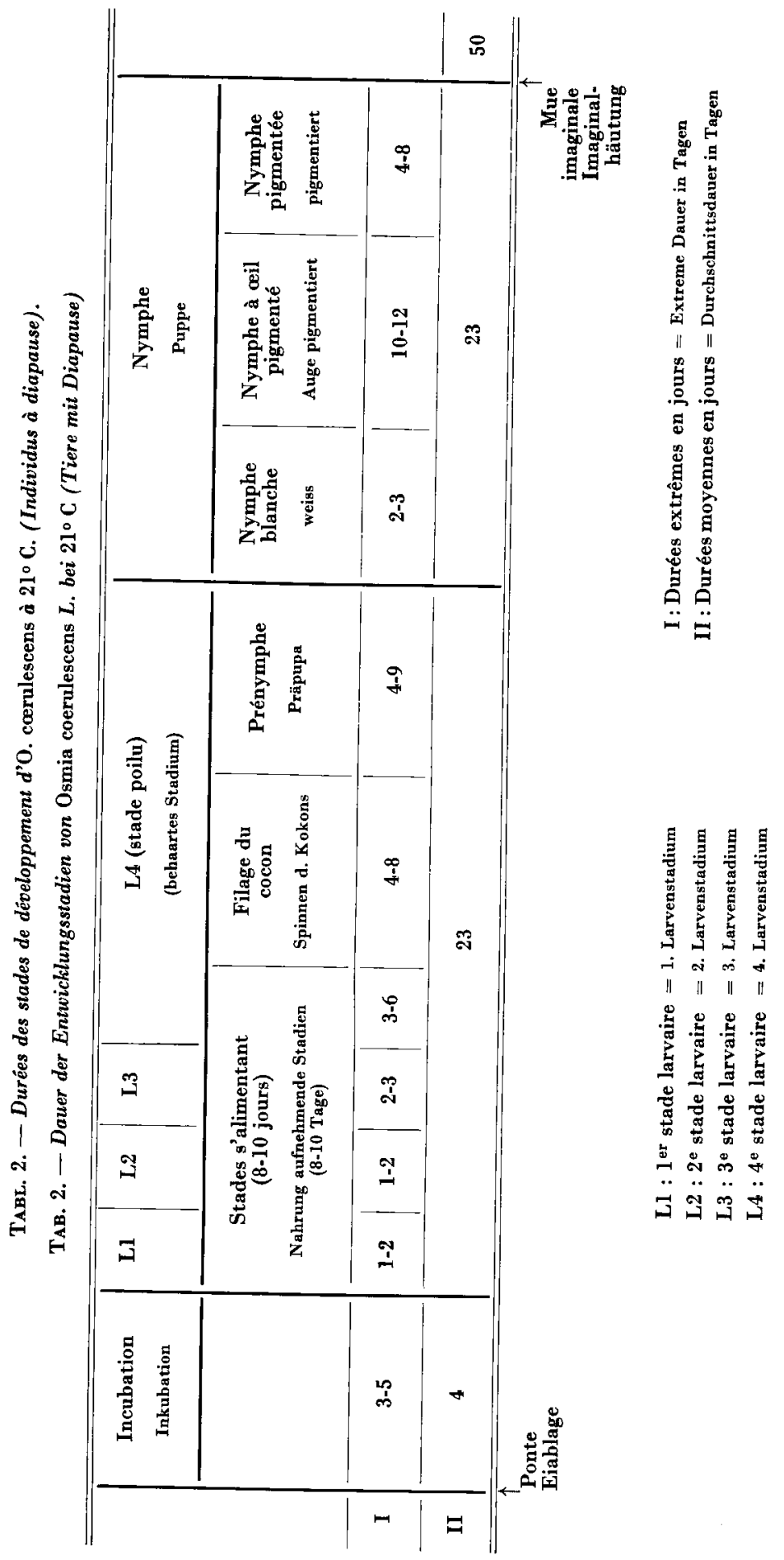


à l'adulte dure environ 7 semaines. A $28^{\circ} \mathrm{C}$, la durée de développement n'est que de 23 jours. $32^{\circ} \mathrm{C}$ est une température critique : les durées du développement s'échelonnent de 26 à 70 jours et la mortalité est très élevée 1 .

A $21^{\circ} \mathrm{C}$ l'incubation demande de 3 à 5 jours (moyenne 4,3). Puis se succèdent 4 stades larvaires, le dernier étant poilu comme chez tous les Megachilidae. Les deux premiers stades durent chacun de 1 à 2 jours, le troisième de 2 à 3 jours et le quatrième de 14 à 20 jours. Ce dernier stade larvaire comprend trois phases : la phase d'alimentation (3-6 jours), la phase de filage du cocon (4-8 jours) et la phase immobile ou prénymphale, précédant la mue nymphale (4-9 jours). La larve s'alimente seulement pendant la première moitié de son existence, soit de 8 à 12 jours. La nymphe, immobile, couchée sur le dos et la tête tournée vers la sortie, comme la prénymphe, est entièrement blanche pendant 2-3 jours, puis ses yeux se pigmentent pendant 10 ou 12 jours, enfin, les mandibules puis le reste du corps noircissent progressivement pendant 4-8 jours jusqu'à la mue imaginale. En résumé, le stade ouf dure 4,3 jours, le stade larve : 23 jours, le stade nymphe : 23 jours. Dans les conditions naturelles, à une température moyenne de $14,7^{\circ} \mathrm{G}$, le développement dure 68 jours; à une température moyenne de $17^{\circ} \mathrm{C}, 55$ jours.

\section{La deuxième génération.}

Lorsque le développement a lieu sous une température constante supérieure à $20^{\circ} \mathrm{C}$, ou bien lorsqu'il a lieu dans les conditions naturelles, on constate qu'une partie des individus arrivés au stade adulte, émerge 5 à 8 jours après la mue imaginale, et qu'une autre partie reste en cocon et ne sort qu'après avoir subi une période de froid. Seuls les oufs pondus en mai donnent tous des insectes de deuxième génération. Toutes les femelles et seulement une partie des mâles issus de la ponte de juin donnent un vol de deuxième génération. Les cufs pondus en juillet, août et septembre donnent tous des osmies à diapause imaginale. En fait, dans la nature, la ponte tardive de la fin d'été donne des individus hibernant à l'état de nymphe.

La première génération nidifie de mai à la mi-juillet. La deuxième génération, qui nidifie de la mi-juillet à la fin-septembre semble beaucoup moins prolifique que la première. Nous avons remarqué que les insectes donnant une deuxième génération, se développent plus rapidement que les individus à diapause $\left(6\right.$ à 7 jours de plus, à $21^{\circ} \mathrm{C}$. Observations portant sur 50 individus).

\section{L'hibernation et la réactivation en conditions contrôlées.}

Les insectes adultes sont élevés à la température constante de $21^{\circ} \mathrm{C}$

1. Les observations concernant les durées de développement à $21^{\circ} \mathrm{C}, 28^{\circ} \mathrm{C}, 32^{\circ} \mathrm{C}$, portent respectivement sur 18, 15, 11 individus. 
pendant le mois qui suit la mue imaginale, puis ils subissent pendant 5 mois une température de 3-4, $\mathrm{C}$. Ils émergent après 20 jours de réactivation à $21^{\circ} \mathrm{C}$.

\section{La mortalité.}

Une partie de la mortalité : $34 \%$ des individus en 1970, $40 \%$ en 1971, n'est due ni au parasitisme, ni à des champignons microscopiques. Elle survient aux stades : œuf, nymphe, et surtout larve. La mortalité due au parasitisme, calculée seulement en 1971, représente $16 \%$ des individus. Quatre espèces parasitent $O$. carulescens dans la région de Lusignan : un coléoptère : Trichodes sp. (Cleridae); un diptère Drosophilidae : Cacoxenus sp. (probablement); deux hyménoptères : Melittobia acasta et Sapyga quinquepunctata (Sapygidae). Les diptères occupent les cellules d'osmie précocement en mai. Les Sapyga, volant en juillet et en août, sont de loin les parasites les plus abondants.

Krombein (1962 et 1967) a trouvé des acariens : Chaetodactylus Krombeini, ainsi que des Melittobia acasta dans des cellules d'O. corulescens. Grandi (1959 et 1961) donne une liste d'hôtes des nids de cette osmie : Coelioxys argentea, Chrysis dichroa, Eurytoma modularis, Kaltenbachia dentata, Hoploryptus signatorius, Miltogramma murinum, Ptinus sexpunctatus.

\section{CONCLUSION}

A la suite de ces observations préliminaires dans la nature et en conditions contrôlées nous devons souligner quelques points importants de comportement et de physiologie d'O. corulescens.

1. Bien que de nombreuses analyses microscopiques de provisions contenues dans les nids témoignent de la récolte fréquente de pollen de luzerne, cette osmie se trouve rarement dans les champs de luzerne. Cette rareté est probablement due, ou bien au manque de sites de nidification naturels, ou bien à leur éloignement des champs. On peut en effet supposer que l'osmie a un rayon d'action assez faible et qu'elle ne s'éloigne guère de son nid lorsque des fleurs attractives assez nombreuses sont proches de son habitat. On peut aussi expliquer l'absence d'O. corulescens dans certaines zones par le fait qu'elle ne trouve pas la plante (Malva moschata) utilisée pour construire son nid.

2. Les plantes compétitrices de la luzerne sont des labiées et surtout d'autres papilionacées (Trèfles, lotier...). Des études ultérieures pourraient conduire à l'analyse détaillée du choix de l'espèce botanique par la femelle. 
3. Une deuxième génération atardive est constituée par les individus issus d'œufs pondus aux mois de mai et juin. Les insectes issus de la ponte des mois de juillet, d'août et septembre restent en diapause et n'émergent que l'année suivante. Les modalités de l'induction de cette diapause, en particulier l'action des photopériodes croissantes et décroissantes, restent à élucider.

4. La préférence de l'espèce pour les cavités forées dans le bois, les Cannes de Provence et les tubes de carton, et la tendance des femelles à nidifier près de l'endroit de leur naissance, laissent entrevoir la possibilité d'accroître et de déplacer des “ pseudocolonies " d'O. corulescens en vue de polliniser la luzerne à graines.

La biologie d'O. ccrulescens offre d'intéressants sujets d'étude dans les domaines de l'éthologie, la physiologie et la recherche appliquée.

Rę̧u pour publication en fêvrier 1972.

Eingegangen im Februar 1972.

\section{REMERCIEMENTS}

Nous tenons à remercier M. Desmier de Chenon (I.N.R.A.) qui a identifié $O$. cœrulescens.

\section{ZUSAMMENFASSUNG}

Verf. berichtet über biologische Beobachtungen an Osmia coerulescens. Obwohl diese Art nur selten in den Luzernefeldern angetroffen wird, bestäubt sie die Luzerne. Sie nistet in grosser Zahl in hohlen Rohrstengeln (ARUNDo L.).

55 Nistanlagen in Rohrstengeln und Papphülsen, die an verschiedenen Plätzen in der Umgebung von Lusignan errichtet wurden, wurden einmal wöchentlich aufgesucht, ausserdem wurden 1970 und 197114 gezeichnete Weibchen im Gewächshaus gehalten. Die Entwicklung vom Ei bis zur Imago wurde im Brutschrank bei konstanten Temperaturen beobachtet.

Die von den Bienen eingetragenen Pollen wurden mikroanalytisch bestimmt und zwar an Proben des sogen. Bienenbrotes, die jeweils zwei Zellen eines Nestes entnommen wurden.

Osmia coerulescens fliegt von Ende April bis Ende September. Während der Monate Mai, Juni, Juli, August herrscht besonders lebhafte Tätigkeit.

Im Gewächshaus schwankt die Lebensdauer der Weibchen zwischen 30 und 60 Tagen. Ein Weibchen legt durchschnittlich 42 Eier; eins bis sechs Eier täglich. Unter natürlichen Bedingungen sind es dagegen nur 1-2 Eier. Das Geschlechterverhältnis erscheint recht variabel; manchmal überwiegen die Männchen, zuzeiten die Weibchen. Zum Nestbau bevorzugt $O$ smia die Ost- und Südfassaden nicht verputzter Gebäude, vor allem von Holzbauten und Löcher von $4 \mathrm{~mm}$ Durchmesser. Die Nester werden linear angelegt. Die Scheidewände und Verschlusspropfen bestehen aus einem grünlichen Mörtel pflanzlichen Ursprungs (Malva moscata Malvaceae). Die durchschnittliche Länge der Zelle beträgt $8,6 \mathrm{~mm}$ bei einem Durchmesser von $4 \mathrm{~mm}$.

Zwei Pflanzenfamilien werden ihres Pollens wegen beflogen : Labiatae und besonders Papilionaceae. Osmia coerulescens gehört zu den Arten, die Pollen auf einer Vielfalt verschie- 
dener Pflanzenfamilien sammeln. Bei ihr geht jedoch die Tendenz dahin, sich auf einige wenige Familien zu beschränken. Die am häufigsten gesammelten Pollen stammen von Trifolium repens, T. pratense, Medicago sativa und Lotus cornulatus. Im Gewächshaus scheint Melilotus alba, der in der Umgebung von Lusignan nicht vorkommt, stärkere Anziehungskraft zu besitzen als die vorerwähnten Pflanzen. Die beflogenen Labiaten sind : Calamintha, Glechoma, Stachys, Lamium, Teucrium, Brunella, Ajuga. Zur Versorgung einer Zelle sind durchschnittlich 20 Sammelflüge $=3$ Arbeitsstunden notwendig. Die Sammelgeschwindigkeit schwankt zwischen 10 und 14 Blüten in der Minute, je nach Pflanze. In der Minute werden 10 Luzerneblüten von einer Osmia « aufgeklinkt 》.

Die Entwicklung vom Ei bis zur Imago dauert 6-7 Wochen bei $21^{\circ} \mathrm{C}$ und ungefähr 23 Tage bei $28^{\circ} \mathrm{C}$. Vier Larvenstadien werden durchlaufen; wie bei allen Megachilidae ist das 4. Stadium behaart. Im Juli erscheint eine zweite, weniger fruchtbare Generation. Aus den von den Weibchen der 1. Generation später als Juni abgelegten Eiern entstehen Imagines, die eine Diapause durchmachen.

Es gelang uns, eine Anzahl von Osmien während der Diapause schlüpfen zu lassen, indem wir sie 5 Monate bei $3-4^{\circ} \mathrm{C}$ überwinterten und danach bei $21^{\circ} \mathrm{C}$ reaktivierten. Die Sterblichkeitsrate, durch unbekannte Ursachen während der Entwicklungszeit bedingt, bewegt sich zwischen 34 und $40 \% .1971$ verursachten Schmarotzer 16,5\% der Verluste. Der am häufigsten vorkommende Schmarotzer ist Sapyga quinquepunctata F. (Sapygidae).

In nicht zu ferner Zukunft dürfte es möglich sein, durch Errichten künstlicher Nistplätze für Osmia coerulescens den Samenertrag der Luzerne zu verbessern, so wie es in U.S.A. mit Megachile rotundata gemacht wurde.

\section{REFERENCES BIBLIOGRAPHIQUES}

Balfour-Browne F. 1922, On the life history of Melittobia acasta Walk. a chalcid parasite of bees and wasps. Parasitology, 14, 349-370.

Benoist R. 1938, Osmia coerulescens L. Rev. sci. du Bourbonn. 84-86.

Benorst R. 193:, Les Osmies de la faune française. Ann. Soc. ent. Fr. 23-60.

Bohant G. E. 1957, Pollination of alfalfa and red clover. Ann. Rev. ent, 2. 355-380.

Cavro E. 1950, Catalogue des hyménoptères du nord et régions limitrophes. II tome, Suppl. Bull. Soc. Ent. Nord de la France, 52.

Chanderer L. 1963, Interspecific competition between two species of Osmia. Proc. North. centr. Branch E.S.A. 18, 30.

Dylewska M. and al. 1970, An attempt of determination of the number of bees ( $\mathrm{Hym}$. Apoidea) needed for adequate pollination of alfalfa. Bull. ent. de Pologne, 40, 371-398.

Ferton C. 1894, Seconde note sur les mcurs de quelques hyménoptères du genre Osmia Panz. principalement de la Provence. Actes Soc. Lin. de Bordeaux, 47, 1-12.

Free J.-B. and Wrtciams I. H. 1970, Preliminary investigations on the occupation of artificial nests by Osmia rufa L. (Hym. Megachilidae). J. appl. Ecol. 7, 559-566.

Friese H. 1911, Apidae I. Das Tierreich 28. Lieferung-Megachilinae. Berlin.

FRIESE H. 1923, Die europäischen Bienen (Apidae). Das Leben und Wirken unserer Blumenwespen. De Gruyter. Berlin U. Leipzig.

Grandi G. 1959, Contributi allo studio degli Imenotteri Aculeata. Boll. Inst. ent. univ. Bologna. 23, 239-292.

Grandi G. 1961, Studi di un entomologo sugli imenotteri superiori. Boll. Inst. ent. univ. Bologna. 295-296.

Hackwell G. 1968, The biology and behavior of the alkali bee Nomia melanderi Cock. Ore. St. univ. Thesis entomology. 
Iuga V. G. et Scobiona X. 1960, Sur quelques apoides pollinisateurs des luzernes. Tr. Mus. Hist. Nat. R.P.R. 2, 245-257.

IugA V. G. 1962, The apoids as pollinators. Tr. Mus. Hist. Nat. \& Gr. Antipa 》 3, 225-237.

Krombein K. V. 1967, Trap nesting wasps and bees life history nests and associates. Wash. D.C. Smithsonian Inst. Press.

Кrombeis K. V. 1962, Natural history of Plummers Island, Maryland-Biological notes on Chaetodactylus Krombeini Bak. a parasitic mite of the megachilid bee, Osmia Lignaria Say. Proc. biol. Soc. Wash. 75, 237-249.

Lecomte J. et Tirgari S. 1962, Sur quelques pollinisateurs des légumineuses fourragères. Ann. Abeille, 8, 83-93.

Lesins K. 1950, Investigations into seed setting of lucerne at Ultuna, Sweden. The Ann. Roy. agric. College of Sweden, 17, 441-483.

Levin M.-D. 1957, Artificial nesting burrows for Osmia lignaria Say. J. of Econ. ent. 50, 506-507.

Linslex E. G. 1958, The ecology of solitary bees. Hilgardia, 27, 543-599.

Linsley E. G. 1946, Insect pollinators of alfalfa in California. J. Econ. ent. 39, 18-29.

Malyshev S. I. 1935, The nesting habits of solitary bees. A comparative study. EOS XI, Lams 3-15.

Marikovskaya T. P. 1968, New data on the biology of some species of social bees (Hymenoptera, Megachilidae) from south-east Kazakhstan. Ent. Rev. 47, 487-491.

Maurizio A. et Louveaux J. 1965, Pollens de plantes mellifères d'Europe; U.G.A.F. 38, Bd Sébastopol. Paris.

MedLer J.-T. 1967, Biology of Osmia in trap nests in Wisconsin. Ann. ent. soc. Amer. 6, 338344.

Moczar J. T. 1961, The distribution of wild bees in lucerne fields of Hungary (Hymenoptera, Apoidea) Ann. Hist. Nat. Mus. Nat. Hung. 53, 452-461.

ObRtel R. et Sedivy J. 1965, Insects pollinating lucerne (Medicago sativa) in CzechoslovakiaZool. listy. 14, 339-348.

Robertson C. 1925, Heterotropic bees. Ecology, 6, 412-436.

Solinas M. et BIn F. 1965, Osservazioni sugli insetti impollinatori delle leguminose foraggere (Medicago sativa e Trifolium pratense) nella pianura padana. Sem. Elette II, 50-128.

Stephen W. P. 1965, Artificial beds for Alkali bee propagation. Bull. Ore, State Univ. 593, 1-20.

TAser J. N. 1971, Influence de la date de la précoupe de la luzerne (Medicago sativa) sur sa pollinisation. Apidologie, 2, 173-195.

Tirgari S. 1968. Étude expérimentale et comparée de la construction et de l'approvisionnement du nid de Melitta leporina Panz. (Hym. Melittidae). Thèse fac. sc. Poitiers.

Tirgari S. 1968, La nidification de Megachile rotundata Fabr. Hym. Megachilidae. Thèse fac. sc. Poitiers.

Walstrom R. L. and Jones P. A. 1968, Alfalfa leaf-cutter bee management for alfalfa pollination in south Dakota. Bull. south Dakota State Univ. 544, 1-12. 